\title{
Feasibility of a Direct-Conversion Method from Magnetic Susceptibility to Relative Electron Density for Radiation Therapy Treatment Planning
}

\author{
Kengo Ito ${ }^{*}$, Noriyuki Kadoya1, Yujiro Nakajima ${ }^{1,2}$, Masahide Saito ${ }^{3}$, Kiyokazu Sato ${ }^{4}$,

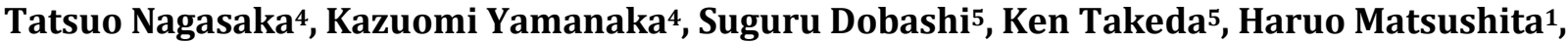 \\ Keiichi Jingu ${ }^{1}$
}

\author{
${ }^{1}$ Department of Radiation Oncology, Tohoku University Graduate School of Medicine, Sendai, Japan \\ ${ }^{2}$ Department of Radiology, Tokyo Metropolitan Komagome Hospital, Tokyo, Japan \\ ${ }^{3}$ Department of Radiology, University of Yamanashi, Chuo-shi, Japan \\ ${ }^{4}$ Department of Radiology, Tohoku University Hospital, Sendai, Japan \\ ${ }^{5}$ Department of Therapeutic Radiology, Tohoku University School of Medicine, Sendai, Japan \\ Email: ^i-kengo@med.tohoku.ac.jp
}

How to cite this paper: Ito, K., Kadoya, N., Nakajima, Y., Saito, M., Sato, K., Nagasaka, T., Yamanaka, K., Dobashi, S., Takeda, K., Matsushita, H. and Jingu, K. (2017) Feasibility of a Direct-Conversion Method from Magnetic Susceptibility to Relative Electron Density for Radiation Therapy Treatment Planning. International Journal of Medical Physics, Clinical Engineering and Radiation Oncology, 6, 252-265.

https://doi.org/10.4236/ijmpcero.2017.63023

Received: May 23, 2017

Accepted: July 15, 2017

Published: July 18, 2017

Copyright (c) 2017 by authors and Scientific Research Publishing Inc.

This work is licensed under the Creative Commons Attribution-NonCommercial International License (CC BY-NC 4.0).

http://creativecommons.org/licenses/by-nc/4.0/

\begin{abstract}
Recently, several institutions have been developing magnetic resonance imaging (MRI)-guided radiotherapy treatment systems. In this study, we examine whether it is possible to perform radiation therapy planning (RTP) using a magnetic susceptibility map obtained using MRI. The head of a healthy volunteer was scanned using dual-energy computed tomography (CT) and MRI. A T2-star-weighted 3D gradient echo-based sequence (GRE) with images taken at four different echo times was acquired using the MRI scanner. The CT images were converted to relative electron density ( $\mathrm{rED}$ ) using a predefined $\triangle \mathrm{CT}$-rED conversion table. $\triangle \mathrm{CT}$ was derived using the energy-subtraction method. The rED map was obtained from a single-linear relationship with the $\triangle \mathrm{CT}$-rED conversion table, whereas the magnetic susceptibility map was obtained from quantitative susceptibility mapping (QSM) via MRI. Subsequently, to obtain the relationship between the magnetic susceptibility and the $\mathrm{rED}$, the rED map was rigidly aligned to the susceptibility map and resampled at the susceptibility map's resolution. Finally, the magnetic susceptibility rED conversion table was obtained via voxel-by-voxel mapping between the two maps. No strong relationship between magnetic susceptibility and rED was obtained in the healthy volunteer's head or in this study. The coefficient correlation between these parameters was 0.0145 . Magnetic susceptibility values may be not able to convert to rED using our proposed method in
\end{abstract}


healthy volunteer's head. In contrast to the magnetic-susceptibility values obtained from the QSM algorithm, which were strongly affected by calcification and iron content, the rED or CT number was not considerably affected by such materials.

\section{Keywords}

Magnetic Susceptibility, Quantitative Susceptibility Mapping, MRI-Based Radiation Therapy Planning

\section{Introduction}

To date, medical facilities have mostly used X-ray-based imaging modalities, such as the On-Board Imaging system, cone-beam computed tomography, Electronic Portal Imaging Device, orthogonal X-ray imaging system (Exac Track, BrainLAB AG, Germany), and Megavoltage computed tomography (Tomotherapy, Accuray Inc, Sunnyvale, CA, USA), as imaging modalities for imageguided radiation therapy (IGRT), which aim to decrease geometric uncertainties during radiotherapy using image guidance [1] [2] [3] [4]. These X-ray-based imaging modalities can easily visualize high-density materials, such as bone and fiducial markers; however, the visualization of low-density materials, such as soft tissues and tumors, is limited. Hence, when we use X-ray-based imaging modalities for IGRT, we often match bone or fiducial markers between planned and obtained images just before the treatment and indirectly obtain tumor positions from matching high-density materials.

Recently, several institutions have been developing magnetic resonance imaging (MRI)-guided radiotherapy treatment systems (i.e., MRgRT or MRIgRT) [5] [6]. The principle behind such systems is using the MRI machine as an imaging modality for IGRT. MR images have an excellent soft-tissue contrast and facilitate a soft-tissue- or tumor-based patient setup with no additional exposure. Furthermore, MRgRT has the potential to reduce the setup margin and adaptive radiation therapy. In order to incorporate MRI images into radiotherapy, several problems and methods for addressing them have been reported by numerous researchers [5] [6]. MRI images have problems with regard to image-distortion, resulting from magnetic-field inhomogeneity induced by a tissue's magnetic susceptibility, and the effect of image distortion has been discussed in previous studies [1] [2] [3] [4] [7] [8] [9]. Furthermore, these previous studies have proposed various correction methods to mitigate such undesirable effects. MRI provides superior image quality for soft tissue delineation compared to computed tomography (CT) and is widely used for target and organ delineation in radiotherapy treatment planning (RTP) [10] [11] [12]. The intensity of CT images is directly related to electron density. Therefore, CT is used for attenuation correction of the doses calculated in RTP. The major challenge in the application of MRI to RTP is the fact that there exists no physical relationship between MR-signal intensities and electron densities. The 
feasibility of MR-based dose calculation has been demonstrated for radiotherapy on different treatment regions using manual segmentation and bulk-density assignment [13] [14]. Conversion from MRI to pseudo-CT images has also been reported using anatomy- and voxel-based methods. Anatomy-based methods use deformable image registration between MRI and CT images to obtain deformed MRI or CT images. The drawback of this method is the uncertainty of the registration [15]. Voxel-based methods can avoid dependence on image registration by direct conversion of MRI signal-intensity values to Hounsfield units (HU) or electron densities [16] [17]. Previous studies on voxel-based methods have used ultrashort echo time sequences to segment tissue into soft tissue, bone, and air.

The abovementioned correction methods and MRI sequences are used for MRI-based RTP. On the other hand, a new quantitative value, magnetic susceptibility, has been utilized in clinical research in addition to the $T_{1}, T_{2}$, and ADC values [18] [19] [20] [21]. Magnetic susceptibility is a physical property of a material that may assist with the detection and quantification of specific biomarkers such as gadolinium, calcium, and iron to assess brain physiology and pathology. A magnetic susceptibility map was generated by deconvolution between the local magnetic field and the dipole kernel. This field was obtained from phase data from gradient echo sequences including not only true signals but also noises and artifacts from hardware and human bodies. The dipole kernel was obtained by approximation, and if deconvolution was performed in the Fourier domain, the coefficients of the dipole kernel become zero on the surface of the cone in $k$-space [22]. Hence, deconvolution between the local magnetic field and the dipole kernel was ill-condition. Recently, however, several approaches have been reported for solving this challenging inverse problem to obtain magnetic susceptibility maps [23] [24] [25] [26] [27].

This study describes our investigation of the feasibility of a voxel-based method for directly converting magnetic susceptibility $(\chi)$ into relative electron density (rED). Chen et al. showed that the total CT number and total susceptibility are strongly correlated in calcification regions [28]. We examined over the entire brain whether there is a correlation between these quantities. Because $\chi$ values are quantitative and if rED can be obtained from $\chi$, MR images can be used instead of CT images in radiotherapy planning.

\section{Materials and Methods}

\subsection{Morphology Enabled Dipole Inversion for Quantitative Susceptibility Mapping}

To obtain the magnetic susceptibility map, we used the morphology enabled dipole inversion (MEDI) approach [24]. MEDI utilizes a constrained $\mathrm{L}_{1}$ normminimization problem,

$$
\min _{\chi}\|M G \chi\|_{1} \quad \text { s.t. }\|W(D \chi-b)\| 2^{2} \leq \varepsilon .
$$

where $M$ is the weighting matrix derived from the gradient of the magnitude image. $G$ denotes the gradient operator, $W$ is a weighting matrix proportional to 
the image magnitude to compensate for the noise variation in the field measurements, and $\varepsilon$ is the noise level. MEDI minimizes the number of voxels that belong to the edges in the magnetic susceptibility map but not the edges in the magnitude image. Therefore, we need to estimate the local magnetic field for QSM using the following formula

$$
B_{L}(\mathbf{r})=B_{T}(\mathbf{r})-B_{B}(\mathbf{r}) .
$$

where $B_{L}(\mathbf{r})$ is the local magnetic field, $B_{T}(\mathbf{r})$ is the total magnetic field, and $B_{B}(\mathbf{r})$ is the background field induced by outside of $W . B_{T}(\mathbf{r})$ is proportional to the measured signal phase $\phi$ and is given as follows:

$$
B_{T}(\mathbf{r})=\phi(\mathbf{r}) /(\gamma \cdot \mathrm{TE}) .
$$

where $\gamma$ is the gyromagnetic ratio of the ${ }^{1} \mathrm{H}$ nucleus and TE is the time to echo in a gradient echo sequence. To avoid aliasing of the frequency distribution and obtain a robust three-dimensional phase map, we performed phase unwrapping [2]. Then, $B_{B}(\mathbf{r})$ could be approximated using projection onto dipole field (PDF) algorithm [29], which allows us to obtain $B_{L}(\mathbf{r})$. The data processing chain for QSM is schematically illustrated in Figure 1.

\subsection{Generation of a Single-Linear Relationship for the Dual-Energy Subtraction $\triangle H U$ - rED Conversion Table}

The conversion of the computed-tomography number (HU) into an electron density relative to water is used in treatment planning for radiation therapy. The HU-rED conversion is performed using tissue substitutes with known electron densities in calibration phantoms [30]. However, because the CT numbers obtained from tissue-attenuation coefficients depend upon both electron densities and effective atomic numbers, the HU-rED conversion table did not express a one-to-one relationship. Hence, we used $\triangle H U-r E D$ conversion method, for which $\triangle H U$ was the energy subtracted HU derived from dual energy computed tomography [31] [32] [33]. This $\triangle H U-r E D$ conversion method can finally generate a single relationship between $\mathrm{HU}(\triangle \mathrm{HU})$ and $\mathrm{rED} . \Delta \mathrm{HU}$ is defined as follows:

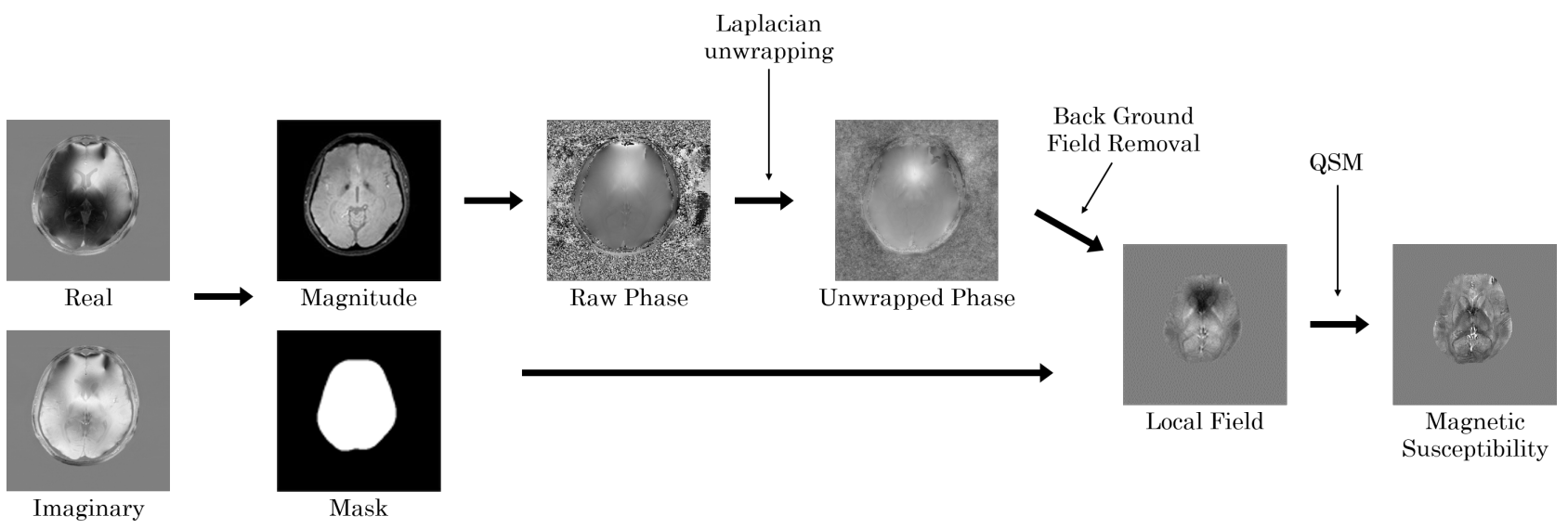

Figure 1. Schematic of the quantitative susceptibility mapping (QSM) algorithm. 


$$
\Delta \mathrm{HU}=(1+\alpha) \mathrm{HU}_{\mathrm{H}}-\alpha \mathrm{HU}_{\mathrm{L}} .
$$

where $\alpha$ is the weighting factor for the subtraction, with $\mathrm{H}$ and $\mathrm{L}$ representing the $\mathrm{CT}$ values from the high and low energy $\mathrm{kV}$ scans, respectively. Thus, we can obtain the single relationship between $\triangle \mathrm{HU}$ and rED given by the following equation:

$$
\mathrm{rED}=a \times \Delta \mathrm{HU} / 1000+b .
$$

where $a$ and $b$ are constant.

To obtain a single relationship between $\triangle H U$ and $\mathrm{rED}$, we scanned a tissue-characterization phantom GAMMEX 467 (Gammex Inc., Middleton, WI) with 14 inserts made of different materials using a CT scanner (LightSpeed RT 16, GE Healthcare, Milwaukee, WI). This scanner could not perform a dualenergy scan, so instead we performed two single-energy scans at different energies. The CT scans were performed with the following parameters: tube voltage $=80$ and $120 \mathrm{kVp}$; tube current $=350 \mathrm{~mA} ;$ FOV $=300 \times 300 \mathrm{~mm}^{2}$; matrix size $=$ $512 \times 512$; slice thickness $=2.5 \mathrm{~mm}$. Figure $2(\mathrm{a})$ and Figure $2(\mathrm{~b})$ show scanned high-kV and low-kV CT images, respectively. This algorithm was implemented in Mathematica (Wolfram Research, Inc., Champaign, IL).

\subsection{Generating the Relationship for the $\chi$-rED Conversion Table}

In-vivo brain imaging of a 27-year-old healthy volunteer was performed at Tohoku University Hospital using a 3.0-tesla MRI scanner (Trio Tim, SIEMENS Medical Systems, Erlangen, Germany) equipped with an 8-channel head coil. The T2-star-weighted images were acquired using a 3D gradient echo-based sequence with four different echo times, as well as the following parameters: number of echoes $=4$; TEs $=9.5,15.4,21.3$, and $27.3 \mathrm{~ms}$, TR $=55 \mathrm{~ms}$, flip angle $=15^{\circ}, \mathrm{FOV}=256 \times 256 \mathrm{~mm}^{2}$, matrix size $=256 \times 256$, slice thickness $=2 \mathrm{~mm}$, number of slices $=64$, pixel bandwidth $=241 \mathrm{~Hz}$. These T2-star-weighted images were used to estimate the phase data. To estimate the local field map $B_{L}(\mathbf{r})$, a Laplacian unwrapping of the phase [34] and a background field-removal PDF algorithm [29] were implemented to process phase data. Then, the magnetic susceptibility map, $\chi(\mathbf{r})$, of the whole brain was reconstructed using the MEDI algorithm. These algorithms were implemented in Mathematica.

In addition, brain-CT images were acquired by dual-energy CT scanning using the same parameters employed in generating the $\triangle H U-r E D$ conversion table. We obtained written informed consent before MR and CT imaging. Then,

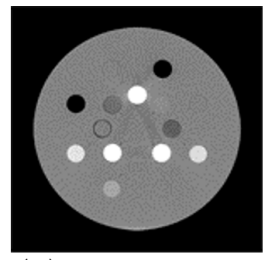

(a)

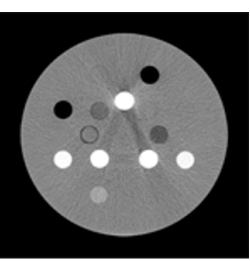

(b)

Figure 2. Scanned (a) high-kV and (b) low-kV computed tomography (CT) images of a tissue-characterization phantom. 

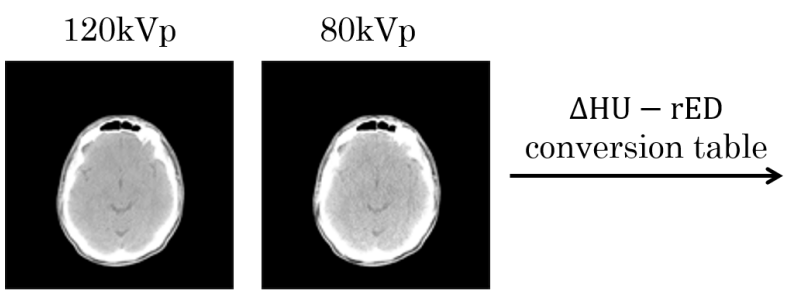

rED map

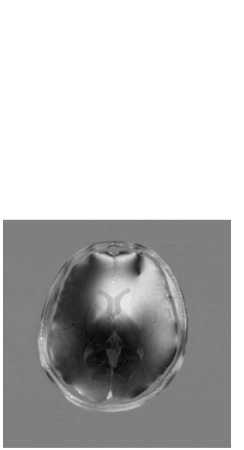

Real

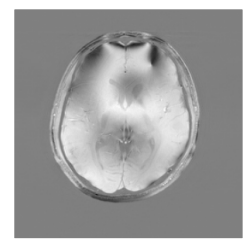

Imaginary

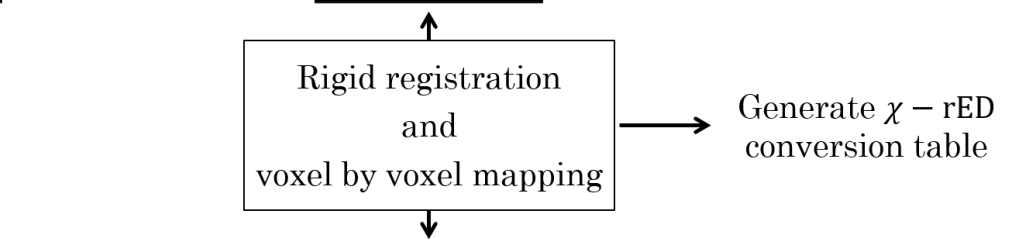

Figure 3. Schematic of the generated $\chi$-rED conversion table.

these CT images were converted to rED using the $\triangle \mathrm{HU}-\mathrm{rED}$ conversion table.

The rED map was rigidly registered with the $\chi$ map and resampled to the magnetic susceptibility map's resolution. Finally, the $\chi$-rED conversion table was obtained via voxel-by-voxel mapping between $\chi$ and rED map. Figure 3 shows a schematic used to generate the $\chi$-rED conversion table.

\section{Results}

\subsection{Dual-Energy-Subtraction $\triangle H U$ - rED Conversion Table}

Figure 4(a) shows the HU-rED and $\triangle H U-r E D$ plot with a straight line fitted using Equation (5). A magnified portion of the soft-tissue region between $\pm 200 \mathrm{HU}$ (the range $\Delta \mathrm{HU}$ ) is shown in Figure 4 (b). The blue and green lines were acquired from high-and low-kV CT scans, respectively. The red line was acquired from Equation (5). The weighting factor, $\alpha$ was set to 1.0, and the coefficient of determination, $r^{2}$, was 0.99 from the fitting process. The obtained a and $b$ values of Equation (5) were 1.16 and 0.98 , respectively. Table 1 lists the average $\mathrm{HU}$ values of each scan as well as the inserted material and the rED and $\triangle H U$ values obtained using Equation (4). In Figure 4, with respect to the general HU-rED curve, the blue and green lines create several segmented linear curves, and we assumed a linear HU-rED relationship in each region. However, the $\triangle H U-r E D$ curve could show a one-to-one linear relationship within a measured $\mathrm{HU}$ value range. Hence, we were able to generate a brain-rED map for voxel-by-voxel mapping between $\chi$ and rED without the effects from several segmented linear HU-rED curves.

\subsection{Magnetic Susceptibility Map from QSM in a Human Brain}

Magnetic susceptibility maps reconstructed with a MEDI-algorithm method are 


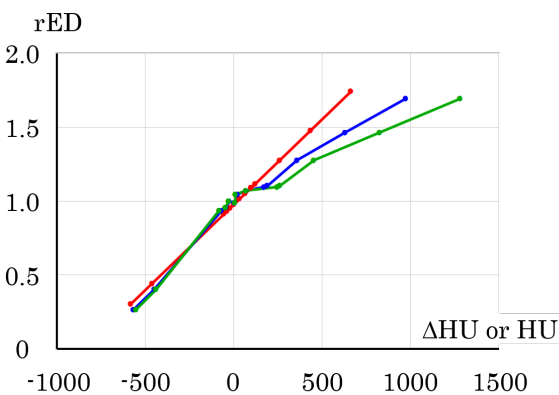

(a)

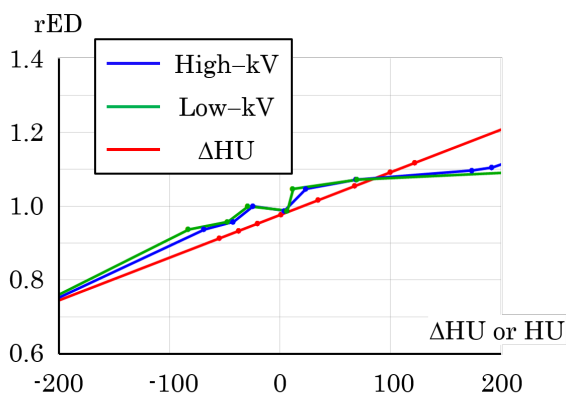

(b)

Figure 4. (a) The blue and green plots show the HU - rED curves obtained from highand low-kV CT scans, respectively. The red plot shows the $\triangle \mathrm{HU}-\mathrm{rED}$ curve obtained from Equations ((6) and (7)). (b) Magnification of the three plots in the soft-tissue region between $\pm 200 \mathrm{HU}$ (the $\Delta \mathrm{HU}$ region).

Table 1. Measured average computed tomography (CT) numbers of each scan and inserted materials and $\triangle \mathrm{HU}$ values obtained from Equation (6). Calculated $\mathrm{rED}$ values were obtained from Equation (7), and referencerED values were nominal.

\begin{tabular}{|c|c|c|c|c|c|}
\hline Materials & High-kV & Low-kV & $\Delta \mathrm{HU}$ & Calculated rED & Reference rED \\
\hline LN-300 Lung & -566.0 & -551.5 & -580.5 & 0.306 & 0.267 \\
\hline LN-450 Lung & -449.0 & -437.6 & -460.4 & 0.444 & 0.404 \\
\hline AP6 Adipose & -68.4 & -82.6 & -54.2 & 0.914 & 0.937 \\
\hline BR-12 Breast & -41.9 & -47.1 & -36.8 & 0.934 & 0.958 \\
\hline Water & -24.3 & -28.7 & -20.0 & 0.953 & 1.000 \\
\hline Solid Water & 4.1 & 6.7 & 1.5 & 0.978 & 0.988 \\
\hline BRN-SR2 Brain & 23.5 & 11.9 & 35.1 & 1.017 & 1.047 \\
\hline LV1 Liver & 69.1 & 70.1 & 68.1 & 1.055 & 1.072 \\
\hline IB Inner Bone & 174.1 & 247.7 & 100.4 & 1.092 & 1.097 \\
\hline B200 Bone Mineral & 192.3 & 262.2 & 122.3 & 1.118 & 1.105 \\
\hline $\mathrm{CB} 2 \%-30 \% \mathrm{CaCO}_{3}$ & 358.2 & 455.1 & 261.4 & 1.278 & 1.278 \\
\hline $\mathrm{CB} 2 \%-50 \% \mathrm{CaCO}_{3}$ & 631.1 & 826.6 & 435.7 & 1.480 & 1.466 \\
\hline SB3 Cortical Bone & 973.7 & 1282.6 & 664.8 & 1.745 & 1.695 \\
\hline
\end{tabular}

shown in Figure 5, which displays the magnitude images (Figure 5(a)) and $\chi$ map (Figure 5(b)). The magnetic susceptibility map represents values in ppm relative to that of water. The streaking artifacts of the dipole kernel were visible as rings in the front of the brain in the $\chi$ map (red arrow in Figure 5(b)). These strong artifacts were observed in some regions, such as the edge of brain and the periphery of sinus, and were caused by the large gradients of the magnetic susceptibilities. To remove the effects of these artifacts from the $\chi-\mathrm{rED}$ conversion table, we defined all values outside of the magnetic susceptibility range of $\pm 1.0 \mathrm{ppm}$ in the $\chi$ map as artifacts and excepted them from voxelby-voxel mapping between $\chi$ and rEDmap. The measured mean magnetic susceptibility values in different brain regions are listed in Table 2, with the results of other reports utilizing the MEDI algorithm for QSM [35]. 
(a)
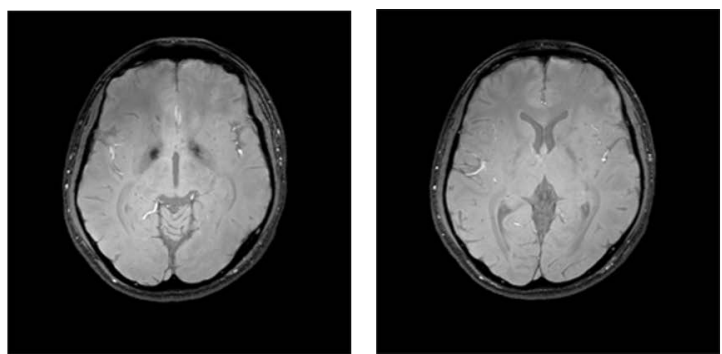

(b)

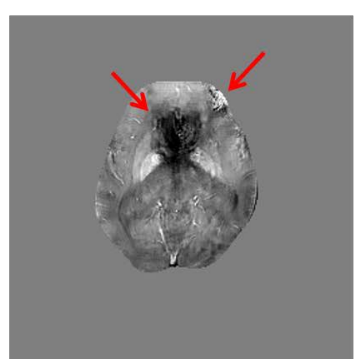

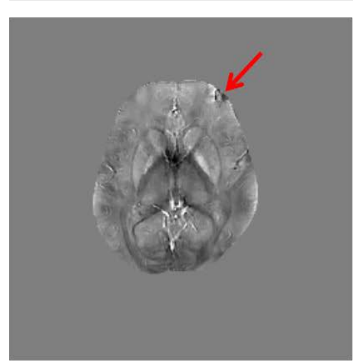
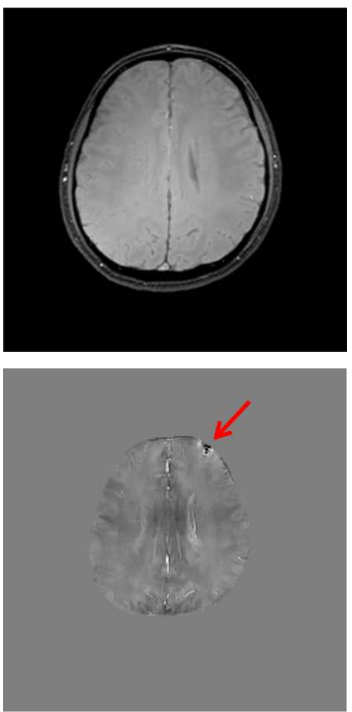

Figure 5. Brain QSM of a healthy volunteer. Top row: Magnitude images. Bottom row: magnetic susceptibility map reconstructed with the morphology enabled dipole inversion (MEDI) algorithm.

Table 2. Comparison of measured mean magnetic susceptibility values in different brain regions in ppm, as obtained in this study and reported in previous studies.

\begin{tabular}{ccc}
\hline & Current study & Liu et al. \\
\hline ppm & Mean \pm SD & Mean \pm SD \\
Globus pallidus & $0.11 \pm 0.10$ & $0.19 \pm 0.02$ \\
Putamen & $0.06 \pm 0.05$ & $0.08 \pm 0.02$ \\
Red nucleus & $0.07 \pm 0.03$ & $0.08 \pm 0.08$ \\
Caudate nucleus & $0.05 \pm 0.04$ & $0.09 \pm 0.02$ \\
\hline
\end{tabular}

Measured values in this study agreed with those of previous studies.

\subsection{Generation of a \\ Magnetic-Susceptibility-to-Relative-Electron-Density Conversion Table}

Figure 6 shows the $\chi$-rED conversion table generated by voxel-by-voxel mapping between $\chi$ and $\mathrm{rED}$. In healthy human brains, the main diamagnetic materials were caused by calcification and visualized as negative signals in QSM. Furthermore, the paramagnetic materials that mainly contributed to the magnetic-susceptibility values were iron contents, which were visualized as positive signal in QSM. The absolute largest magnetic-susceptibility values were in proportion to calcification or iron content, and those close to zero ppm were principally indicated by cerebrospinal fluid and brain matter containing microscopic diamagnetic or paramagnetic materials. The correlation coefficient between magnetic susceptibility and relative electron density was 0.0145 .

\section{Discussion}

We investigated the feasibility of a method for generating a rED map from a $\chi$ 


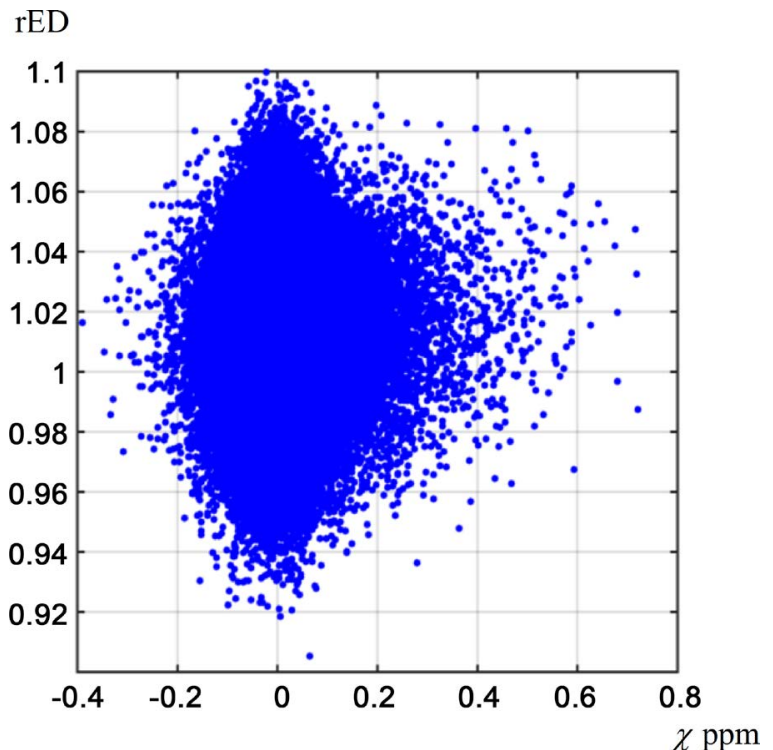

Figure 6 . The $\chi$-rED conversion table generated by voxel-by-voxel mapping between $\chi$ and rED.

map obtained using MRI. In this study, we scanned the head of a healthy volunteer and attempted to convert the brain magnetic susceptibility to relative electron density. The magnetic susceptibility measured by QSM is determined by the molecular composition within an imaging voxel. Further, QSM can visualize the digitized true magnetic susceptibility and MR signals are generated by protons. Hence, magnetic susceptibility values obtained using QSM are influenced by the spatial variation in proton density and composition [36]. Furthermore, the number of electrons is proportional to the number of protons.MR quantitative values, such as magnetic susceptibility, $\mathrm{T} 1$, and $\mathrm{T} 2$, as well as magnitude images, such as T1- and T2-weighted images, are influenced by proton density. In this study, we evaluated the feasibility of QSM and magnetic susceptibility, which are relatively new techniques, as well as quantitative values as an imaging modality to obtain rED for MRI to perform only RTP. However, magnetic susceptibility and QSM contrast are determined by elements with strong susceptibilities, such as calcium and iron, and the influence of electrons on magnetic susceptibility and QSM contrast is very weak. Hence, no strong relationship between magnetic susceptibility and rED was obtained in the healthy volunteer's head or in this study. If we had scanned avolunteer's head containing calcification and attempted to convert magnetic susceptibility values to relative electron density, we might have been able to obtain a different relationship. The different brain regions, such as the globus pallidus, putamen, red nucleus, and caudate nucleus, display different magnetic susceptibility values because each region has a different iron content [26] [37]. When we used some parameters reported in previous research [37], we were able to obtain the absolute iron content of each voxel from the $\chi$ map. Furthermore, we may be able to obtain the dose-to-medium reflected absolute iron content at each voxel. However, because the difference in the absolute iron content between each voxel is minute, even if we adjust the 
dose distribution to the absolute iron content, the small difference will be of little clinical significance. We obtained the $\chi$ map utilizing the optimization technique and multiecho gradient echo sequence. If we change the optimization or sequence parameters, we may obtain different $\chi$ maps. It is important to use the same optimization and sequence parameters for the repeatability of the data.

In QSM, diamagnetically and paramagnetically susceptible materials can be visualized as negative and positive, respectively. In previous studies, when we performed MRI-based radiotherapy planning or attenuation correction, GREmagnitude images were often utilized to generate pseudo-CT images or contourbased segmented MRI images [38] [39]. GRE-magnitude images visualize diamagnetic calcification and paramagnetic cerebral hemorrhaging as a positive signal. Hence, we may fail to find appropriate segmentation or attenuation corrections when we use only GRE-magnitude images. However, if we utilize magnetic susceptibility map to make contour, we may be able to appropriate segmentation and attenuation corrections. In this study, we examined only one healthy volunteer's head, and the brain tissue excluding the bone was evaluated to study the relationship between $\chi$ and rED. This is because strong artifacts were visualized on the brain surface in the $\chi$ map. If we scan the body trunk or pelvic regions, because bones such as the pelvic bone and femurs are located inside the body enough to avoid the artifacts, we may be able to obtain other relationships between $\chi$ and rED.

We utilized a $\triangle H U$-rED conversion method to obtain a rED map from dual-energy CT scans. The generated $\triangle \mathrm{HU}-\mathrm{rED}$ conversion table has a high linearity. Our proposed $\chi$-rED conversion method has especial importance in the soft-tissue region (Figure 4(b)). If we used a general HU-rED conversion method, the $\chi$-rED conversion table was subjected to the undesired effects of the segmented linear relationship the soft-tissue region. However, this undesired effect did not appear when we utilized the $\triangle H U-$ rED conversion table to generate the $\chi$-rED conversion table because this method offers high linearity in this region. The obtained $\triangle H U-r E D$ conversion table contained residual errors between calculated and reference rED. In previous studies, researchers scanned a tissue characterization phantom with various scan parameters, particularly tube voltage, e.g., $80-140 \mathrm{kVp}, 80-140 \mathrm{kVp}$ with Sn filter, and $100-140 \mathrm{kVp}$ [31] [32], and generated the optimum $\Delta \mathrm{HU}-\mathrm{rED}$ conversion table. In this study, we scanned a phantom with clinical scan parameters (80 and $120 \mathrm{kVp}$ ) and obtained the $\triangle \mathrm{HU}-\mathrm{rED}$ conversion table without investigating the optimum scan parameters. If the $\triangle \mathrm{HU}-\mathrm{rED}$ conversion table is generated with optimum scan parameters, the residual error between calculated and reference $\mathrm{rED}$ may be smaller.

Some artifacts were observed in the magnetic susceptibility map in the peripheral sinus and at the edge of brain (Figure 5). In this study, we scanned the head of a healthy volunteer with MRI using the imaging parameters TEs $=9.5$, $15.4,21.3$, and $27.3 \mathrm{~ms}$. The TE value of $9.5 \mathrm{~ms}$ is relatively longer than those used in previous studies [21] [24] [35]. The QSM algorithms utilized the local 
magnetic field obtained from the phase data. Hence, obtaining accurate phase data was very important. However, our first scan time was $9.5 \mathrm{~ms}$; this long first TE caused undesired dephasing and affected estimation of the local magnetic field. As a result, some artifacts were observed in the large-magnetic-susceptibility-gradient regions, such as the peripheral sinus and the edge of the brain. In spite of the presence of artifacts, the measured magnetic-susceptibility values of several deep-gray-matter regions in this study appeared approximately equivalent to their values in the literature [35], and our QSM algorithm performed suitably at generating the magnetic susceptibility map. The difference in the magnetic-susceptibility values between this and the previous study may have been caused by some imaging parameters, such as voxel size, NEX, bandwidth, and the number of subjects. In contrast to our study that scanned one volunteer, the previous study scanned nine volunteers.

\section{Conclusion}

In this study, we attempted to generate a conversion table between magnetic susceptibility and relative electron density using the QSM algorithm and dualenergy CT scans. Correlations between magnetic susceptibility and relative electron density or CT number were not observed in the healthy volunteer's head using our proposed method. Although magnetic susceptibility was strongly affected by iron content and calcification, neither the relative electron density nor the CT number were similarly affected. We evaluated our proposed method using only one healthy volunteer's head. If we scanned more patients or other anatomical sites, such as the body trunk and pelvic regions, we may be able to obtain other relationships that were not acquired in this study; we aim to perform this in our future studies.

\section{Acknowledgements}

This work was supported by a JSPS KAKENHI Grant Number JP 15K19198.

\section{References}

[1] Crijns, S.P., Raaymakers, B.W. and Lagendijk, J.J. (2011) Real-Time Correction of Magnetic Field Inhomogeneity-Induced Image Distortions for MRI-Guided Conventional and Proton Radiotherapy. Physics in Medicine \& Biology, 56, 289-297. https://doi.org/10.1088/0031-9155/56/1/017

[2] Cusack, R. and Papadakis, N. (2002) New Robust 3-D Phase Unwrapping Algorithms: Application to Magnetic Field Mapping and Undistorting Echoplanar Images. Neuroimage, 16, 754-764.

[3] Irarrazabal, P., Meyer, C.H., Nishimura, D.G. and Macovski, A. (1996) Inhomogeneity Correction Using an Estimated Linear Field Map. Magnetic Resonance in Medicine, 35, 278-282. https://doi.org/10.1002/mrm.1910350221

[4] Moerland, M.A., Beersma, R., Bhagwandien, R., Wijrdeman, H.K. and Bakker, C.J. (1995) Analysis and Correction of Geometric Distortions in 1.5 T Magnetic Resonance Images for Use in Radiotherapy Treatment Planning. Physics in Medicine \& Biology, 40, 1651-1654. https://doi.org/10.1088/0031-9155/40/10/007 
[5] Prabhakar, R., Julka, P.K., Ganesh, T., Munshi, A., Joshi, R.C. and Rath, G.K. (2007) Feasibility of Using MRI Alone for 3D Radiation Treatment Planning in Brain Tumors. Japanese Journal of Clinical Oncology, 37, 405-411. https://doi.org/10.1093/jjco/hym050

[6] Pasquier, D., Betrouni, N., Vermandel, M., Lacornerie, T., Lartigau, E. and Rousseau, J. (2006) MRI Alone Simulation for Conformal Radiation Therapy of Prostate Cancer: Technical Aspects. Proceedings of the 28th IEEE EMBS Annual International Conference, New York City, 30 Aug.-3 Sept. 2006, 160-163.

https://doi.org/10.1109/IEMBS.2006.260341

[7] Reber, P.J., Wong, E.C., Buxton, R.B. and Frank, L.R. (1998) Correction of Off Resonance-Related Distortion in Echo-Planar Imaging Using EPI-Based Field Maps. Magnetic Resonance in Medicine, 39, 328-330.

https://doi.org/10.1002/mrm.1910390223

[8] Stanescu, T., Wachowicz, K. and Jaffray, D.A. (2012) Characterization of Tissue Magnetic Susceptibility-Induced Distortions for MRIgRT. Medical Physics, 39, 7185-7193. https://doi.org/10.1118/1.4764481

[9] Kadah, Y.M. and Hu, X. (1997) Simulated Phase Evolution Rewinding (SPHERE): A Technique for Reducing B0 Inhomogeneity Effects in MR Images. Magnetic Resonance in Medicine, 38, 615-627. https://doi.org/10.1002/mrm.1910380416

[10] Potter, R., Heil, B., Schneider, L., Lenzen, H., al-Dandashi, C. and Schnepper, E. (1992) Sagittal and Coronal Planes from MRI for Treatment Planning in Tumors of Brain, Head and Neck: MRI Assisted Simulation. Radiotherapy Oncology, 23, 127 130. https://doi.org/10.1016/0167-8140(92)90344-T

[11] Rasch, C., Barillot, I., Remeijer, P., Touw, A., Van, H.M. and Lebesque, J.V. (1999) Definition of the Prostate in CT and MRI: A Multi-Observer Study. International Journal of Radiation Oncology, Biology, Physics, 43, 57-66. https://doi.org/10.1016/S0360-3016(98)00351-4

[12] Khoo, V.S., Adams, E.J., Saran, F., Bedford, J.L., Perks, J.R., Warrington, A.P. and Brada, M. (2000) A Comparison of Clinical Target Volumes Determined by CT and MRI for the Radiotherapy Planning of Base of Skull Meningiomas. International Journal of Radiation Oncology, Biology, Physics, 46, 1309-1317. https://doi.org/10.1016/S0360-3016(99)00541-6

[13] Jonsson, J.H., Karlsson, M.G., Karlsson, M. and Nyholm, T. (2010) Treatment Planning Using MRI Data: An Analysis of the Dose Calculation Accuracy for Different Treatment Regions. Radiation Oncology, 5, 62.

https://doi.org/10.1186/1748-717X-5-62

[14] Chen, L., Price, R.A.J., Wang, L., Li, J., Qin, L., McNeeley, S., Ma, C.M., Freedman, G.M. and Pollack, A. (2004) MRI-Based Treatment Planning for Radiotherapy: Dosimetric Verification for Prostate IMRT. International Journal of Radiation Oncology, Biology, Physics, 60, 636-647. https://doi.org/10.1016/j.ijrobp.2004.05.068

[15] Hofmann, M., Steinke, F., Scheel, V., Charpiat, G., Farquhar, J., Aschoff, P., Brady, M., Scholkopf, B. and Pichler, B.J. (2008) MRI-Based Attenuation Correction for PET/MRI: A Novel Approach Combining Pattern Recognition and Atlas Registration. Journal of Nuclear Medicine, 49, 1875-1883. https://doi.org/10.2967/jnumed.107.049353

[16] Keereman, V., Fierens, Y., Broux, T., Deene, Y., Lonneux, M. and Vandenberghe, S. (2010) MRI-Based Attenuation Correction for PET/MRI Using Ultrashort Echo Time Sequences. Journal of Nuclear Medicine, 51, 812-818. https://doi.org/10.2967/jnumed.109.065425

[17] Johansson, A., Karlsson, M. and Nyholm, T. (2011) CT Substitute Derived from 
MRI Sequences with Ultrashort Echo Time. Medical Physics, 38, 2708-2714. https://doi.org/10.1118/1.3578928

[18] Acosta-Cabronero, J., Williams, G.B., Cardenas-Blanco, A., Arnold, R.J., Lupson, V. and Nestor, P.J. (2013) In Vivo Quantitative Susceptibility Mapping (QSM) in Alzheimer's Disease. PLoS One, 8, e81093. https://doi.org/10.1371/journal.pone.0081093

[19] Acosta-Cabronero, J., Betts, M.J., Cardenas-Blanco, A., Yang, S. and Nestor, P.J. (2016) In Vivo MRI Mapping of Brain Iron Deposition across the Adult Lifespan. Journal of Neuroscience, 36, 364-374. https://doi.org/10.1523/JNEUROSCI.1907-15.2016

[20] Zhang, J., Liu, T., Gupta, A., Spincemaille, P., Nguyen, T.D. and Wang, Y. (2015) Quantitative Mapping of Cerebral Metabolic Rate of Oxygen (CMRO2) Using Quantitative Susceptibility Mapping (QSM). Magnetic Resonance in Medicine, 74, 945-952. https://doi.org/10.1002/mrm.25463

[21] Azuma, M., Hirai, T., Yamada, K., Yamashita, S., Ando, Y., Tateishi, M., Iryo, Y., Yoneda, T., Kitajima, M., Wang, Y. and Yamashita, Y. (2016) Lateral Asymmetry and Spatial Difference of Iron Deposition in the Substantia Nigra of Patients with Parkinson Disease Measured with Quantitative Susceptibility Mapping. American Journal of Neuroradiology, 37, 782-788. https://doi.org/10.3174/ajnr.A4645

[22] Liu, C., Li, W., Tong, K.A., Yeom, K.W. and Kuzminski, S. (2015) SusceptibilityWeighted Imaging and Quantitative Susceptibility Mapping in the Brain. Journal of Magnetic Resonance Imaging, 42, 23-41. https://doi.org/10.1002/jmri.24768

[23] Wharton, S., Schafer, A. and Bowtell, R. (2010) Susceptibility Mapping in the Human Brain Using Threshold-Based K-Space Division. Magnetic Resonance in Medicine, 63, 1292-1304. https://doi.org/10.1002/mrm.22334

[24] Liu, J., Liu, T., Rochefort, L., Ledoux, J., Khalidov, I., Chen, W., Tsiouris, A.J., Wisnieff, C., Spincemaille, P., Prince, M.R. and Wang, Y. (2012) Morphology Enabled Dipole Inversion for Quantitative Susceptibility Mapping Using Structural Consistency between the Magnitude Image and the Susceptibility Map. Neuroimage, 59, 2560-2568. https://doi.org/10.1016/j.neuroimage.2011.08.082

[25] Khabipova, D., Wiaux, Y., Gruetter, R. and Marques, J.P. (2015) A Modulated Closed Form Solution for Quantitative Susceptibility Mapping-A Thorough Evaluation and Comparison to Iterative Methods Based on Edge Prior Knowledge. Neuroimage, 107, 163-174. https://doi.org/10.1016/j.neuroimage.2014.11.038

[26] Wharton, S. and Bowtell, R. (2010) Whole-Brain Susceptibility Mapping at High Field: A Comparison of Multiple- and Single-Orientation Methods. Neuroimage, 53, 515-525. https://doi.org/10.1016/j.neuroimage.2010.06.070

[27] Lim, I.A., Li, X., Jones, C.K., Farrell, J.A., Vikram, D.S. and Van, Z.P.C. (2014) Quantitative Magnetic Susceptibility Mapping without Phase Unwrapping Using WASSR. Neuroimage, 86, 265-279. https://doi.org/10.1016/j.neuroimage.2013.09.072

[28] Chen, W., Zhu, W., Kovanlikaya, I., Kovanlikaya, A., Liu, T., Wang, S., Salustri, C. and Wang, Y. (2014) Intracranial Calcifications and Hemorrhages: Characterization with Quantitative Susceptibility Mapping. Radiology, 270, 496-505. https://doi.org/10.1148/radiol.13122640

[29] Liu, T., Khalidov, I., Rochefort, L., Spincemaille, P., Liu, J., Tsiouris, A.J. and Wang, Y. (2011) A Novel Background Field Removal Method for MRI Using Projection onto Dipole Fields (PDF). NMR in Biomedicine, 24, 1129-1136. https://doi.org/10.1002/nbm.1670

[30] Schneider, U., Pedroni, E. and Lomax, A. (1996) The Calibration of CT Hounsfield Units for Radiotherapy Treatment Planning. Physics in Medicine and Biology, 41, 
111-124. https://doi.org/10.1088/0031-9155/41/1/009

[31] Saito, M. (2012) Potential of Dual-Energy Subtraction for Converting CT Numbers to Electron Density Based on a Single Linear Relationship. Medical Physics, 39, 2021-2030. https://doi.org/10.1118/1.3694111

[32] Tsukihara, M., Noto, Y., Hayakawa, T. and Saito, M. (2013) Conversion of the Energy-Subtracted CT Number to Electron Density Based on a Single Linear Relationship: An Experimental Verification Using a Clinical Dual-Source CT Scanner. Physics in Medicine and Biology, 58, N135-N144.

[33] Ogata, T., Ueguchi, T., Yagi, M., Yamada, S., Tanaka, C., Ogihara, R., Isohashi, F., Yoshioka, Y., Tomiyama, N., Ogawa, K. and Koizumi, M. (2013) Feasibility and Accuracy of Relative Electron Density Determined by Virtual Monochromatic CT Value Subtraction at Two Different Energies Using the Gemstone Spectral Imaging. Radiation Oncology, 8, 83. https://doi.org/10.1186/1748-717X-8-83

[34] Schofield, M.A. and Zhu, Y. (2003) Fast Phase Unwrapping Algorithm for Interferometric Applications. Optics Letters, 28, 1194-1196. https://doi.org/10.1364/OL.28.001194

[35] Liu, T., Liu, J., Rochefort, L., Spincemaille, P., Khalidov, I., Ledoux, J.R. and Wang, Y. (2011) Morphology Enabled Dipole Inversion (MEDI) from a Single-Angle Acquisition: Comparison with COSMOS in Human Brain Imaging. Magnetic Resonance in Medicine, 66, 777-783. https://doi.org/10.1002/mrm.22816

[36] Liu, C., Wei, H., Gong, N.J., Cronin, M., Dibb, R. and Decker, K. (2015) Quantitative Susceptibility Mapping: Contrast Mechanisms and Clinical Applications. Tomography, 1, 3-17.

[37] Schweser, F., Deistung, A., Lehr, B.W. and Reichenbach, J.R. (2011) Quantitative Imaging of Intrinsic Magnetic Tissue Properties Using MRI Signal Phase: An Approach to in Vivo Brain Iron Metabolism? Neuroimage, 54, 2789-2807. https://doi.org/10.1016/j.neuroimage.2010.10.070

[38] Lee, Y.K., Bollet, M., Charles-Edwards, G., Flower, M.A., Leach, M.O., McNair, H., Moore, E., Rowbottom, C. and Webb, S. (2003) Radiotherapy Treatment Planning of Prostate Cancer Using Magnetic Resonance Imaging Alone. Radiotherapy and Oncology, 66, 203-216. https://doi.org/10.1016/S0167-8140(02)00440-1

[39] Juttukonda, M.R., Mersereau, B.G., Chen, Y., Su, Y., Rubin, B.G., Benzinger, T.L., Lalush, D.S. and An, H. (2015) MR-Based Attenuation Correction for PET/MRI Neurological Studies with Continuous-Valued Attenuation Coefficients for Bone through a Conversion from R2 to CT-Hounsfield Units. Neuroimage, 112, 160-168. https://doi.org/10.1016/j.neuroimage.2015.03.009 
Submit or recommend next manuscript to SCIRP and we will provide best service for you:

Accepting pre-submission inquiries through Email, Facebook, LinkedIn, Twitter, etc. A wide selection of journals (inclusive of 9 subjects, more than 200 journals)

Providing 24-hour high-quality service

User-friendly online submission system

Fair and swift peer-review system

Efficient typesetting and proofreading procedure

Display of the result of downloads and visits, as well as the number of cited articles Maximum dissemination of your research work

Submit your manuscript at: http://papersubmission.scirp.org/

Or contact ijmpcero@scirp.org 